\title{
Poincaré duality for $p$-adic Lie groups
}

\author{
Annette Huber
}

\begin{abstract}
We establish Poincaré duality for continuous group cohomology of $p$-adic Lie groups with rational coefficients and compare integral structures under this duality.
\end{abstract}

Mathematics Subject Classification (2010). Primary 22E41, Secondary 18H10, 22E05.

Keywords. $p$-adic Lie groups, group cohomology, duality.

\section{Introduction}

Let $p$ be a prime and $G$ a $p$-adic Lie group of dimension $d$. Lazard proved that a small enough open subgroup $U$ of $G$ is a Poincaré group, i.e., Poincaré duality holds for continuous group cohomology of $U$ with finite coefficients. This implies that there is a canonical isomorphism $\eta: H^{d}\left(U, D_{\mathbb{Q}_{p}}\right) \cong \mathbb{Q}_{p}$ where $D$ is the dualizing module (see Section 2 for more details). It is compatible under corestriction. We extend this duality to any $p$-adic Lie group $G$. Our first aim is to deduce Poincaré duality for group cohomology with coefficients in $\mathbb{Q}_{p}$-vector spaces.

Theorem 1.1. Let $G$ be a p-adic Lie group of dimension $d$ and $V$ be a finite dimensional $\mathbb{Q}_{p}$-vector space with a continuous operation of $G$. Let $V^{\vee}=$ $\operatorname{Hom}_{\mathbb{Q}_{p}}\left(V, D_{\mathbb{Q}_{p}}\right)$ its dual. Then the natural cup-product pairing

$$
<\cdot, \cdot>: H^{i}(G, V) \times H^{d-i}\left(G, V^{\vee}\right) \rightarrow H^{d}\left(G, D_{\mathbb{Q}_{p}}\right) \cong \mathbb{Q}_{p}
$$

is perfect.

Moreover, we are able to control integral structures under this pairing. In the simplest case $V=\mathbb{Q}_{p}$, the statement is as follows:

Theorem 1.2. Under the pairing of Theorem 1.1 for $V=\mathbb{Q}_{p}$

$$
H^{0}\left(G, \mathbb{Q}_{p}\right) \times H^{d}\left(G, D_{\mathbb{Q}_{p}}\right) \rightarrow \mathbb{Q}_{p}
$$

we have

$$
<1, \eta^{-1}(1)>=1
$$


We would like to emphasize that the statement is not tautological. It needs and has a proof.

This type of result is needed in results on the Tamagawa number conjecture of Bloch and Kato (with $G$ a local or global Galois group) or on integral versions of the Lazard isomorphism. Our main interest was to justify Definition 1.3.2 in [HK], see loc.cit. Remark 1.3.3.

Theorem 1.1 is not surprising and was certainly known to experts. However, it does not seem to be stated in the literature.

Symonds and Weigel in [SW] systematically study cohomology for compact $p$-adic analytic $G$. Their integral Poincaré duality takes the form of an isomorphism betwenn cohomology and homology of Poincaré groups in loc. cit. Theorem 4.4.3. It is not immediate to us how the above Theorem $1.1-\mathrm{a}$ duality with rational coefficients - follows from this.

There are two more technical differences worth pointing out. Symonds and Weigel reprove Lazard's result for finite coefficients on the way, whereas we use it as a starting point. On the other hand, we make systematic use of triangulated categories and exact functors between them, whereas they stay in the setting of abelian or exact categories and study $\delta$-functors.

In my approach, the above results say less about the rational cohomology but more about triangulated $\mathbb{Z}_{p}$-duality and its comparison with $\mathbb{Q}_{p} / \mathbb{Z}_{p}$-duality. Rational Poincaré duality is not addressed by Nekovar in $[\mathrm{N}]$. However, the $\mathbb{Z}_{p}$-duality statement is contained as the arrow $\mathcal{D}$ in his 'duality diagram' $[\mathrm{N}]$ 5.5.4 after identifying all terms. We found it easier to go through the argument directly than to deduce it from the general machine.

\section{Poincaré groups and Pontrjagin duality}

Let $p$ be a fixed prime and $G$ a Poincaré group of dimension $d$. This means ([Se] I $\S 4$, Definition 4.5) that $G$ is a pro- $p$-group such that for continuous group cohomology we have:

1. $H^{s}\left(G, \mathbb{F}_{p}\right)$ is finite for all $s$

2. $H^{d}\left(G, \mathbb{F}_{p}\right) \cong \mathbb{F}_{p}$

3. Cup-product induces a non-degenerate bilinear pairing

$$
H^{s}\left(G, \mathbb{F}_{p}\right) \times H^{d-s}\left(G, \mathbb{F}_{p}\right) \rightarrow \mathbb{F}_{p}
$$

By [Se] I $\S 4$ this implies Pontrjagin duality for cohomology with $p$-power torsion coefficients. The dualizing module (denoted $I$ in [Se]) is of the form $D \otimes \mathbb{Q}_{p} / \mathbb{Z}_{p}$ for a certain $\mathbb{Z}_{p}$-module $D$ free of rank 1 with continuous operation of $G$. By taking limits over duality with torsion coefficients, we get a perfect pairing

$$
H^{s}(G, M) \times H^{d-s}\left(G, \operatorname{Hom}_{\mathbb{Z}_{p}}\left(M, D \otimes \mathbb{Q}_{p} / \mathbb{Z}_{p}\right)\right) \rightarrow \mathbb{Q}_{p} / \mathbb{Z}_{p}
$$

for any finitely generated $\mathbb{Z}_{p}$-module with continuous operation of $G$. We refer to this pairing as Pontrjagin duality. 
By the universal property of $D \otimes \mathbb{Q}_{p} / \mathbb{Z}_{p}$ there is a distinguished isomorphism

$$
\text { can : } H^{d}\left(G, D \otimes \mathbb{Q}_{p} / \mathbb{Z}_{p}\right) \cong \mathbb{Q}_{p} / \mathbb{Z}_{p} \text {. }
$$

By Pontrjagin duality for $M=D$ and $s=d$, this induces also an isomorphism

$$
\begin{aligned}
\eta: H^{d}(G, D) \cong \operatorname{Hom}_{\mathbb{Z}_{p}}\left(H^{0}\left(G, \mathbb{Q}_{p} / \mathbb{Z}_{p}\right), \mathbb{Q}_{p} / \mathbb{Z}_{p}\right) \\
\quad=\operatorname{Hom}_{\mathbb{Z}_{p}}\left(\mathbb{Q}_{p} / \mathbb{Z}_{p}, \mathbb{Q}_{p} / \mathbb{Z}_{p}\right)=\mathbb{Z}_{p}
\end{aligned}
$$

Let $U \subset G$ be an open subgroup. Then there are the natural restriction and corestriction maps

$$
\text { res : } H^{i}(G, M) \rightarrow H^{i}(U, M) \quad \text { cores }: H^{i}(U, M) \rightarrow H^{i}(G, M)
$$

$($ see $[\mathrm{NSW}]$ Ch.1 $\S 5)$. We have coresores $=(G: U)$ (loc.cit. Ch. I $\S 5$ Corollary 1.5.7).

Lemma 2.1. $\eta_{G} \circ$ cores $=\eta_{U}$.

Proof. By [Se] I Prop. 30 (4), we have $\operatorname{can}_{G}=\operatorname{can}_{U}$ ocores. By the projection formula [NSW] Ch. I $\S 5$, Proposition 1.5 .3 we have for all $x \in H^{0}\left(U, \mathbb{Q}_{p} / \mathbb{Z}_{p}\right)$ and $y \in H^{d}(G, D)$ the relation

$$
\operatorname{cores}(\operatorname{res}(x) \cup y)=x \cup \operatorname{cores}(y)
$$

and hence

$$
\operatorname{can}_{G}(\operatorname{res}(x) \cup y)=\operatorname{can}_{U}(x \cup \operatorname{cores} y)
$$

This means that under the isomorphism

$$
H^{d}(G, D) \rightarrow \operatorname{Hom}_{\mathbb{Z}_{p}}\left(H^{0}\left(G, \mathbb{Q}_{p} / \mathbb{Z}_{p}\right), \mathbb{Q}_{p} / \mathbb{Z}_{p}\right)
$$

corestriciton on the left is dual to restriction on the right. Obviously res : $H^{0}\left(G, \mathbb{Q}_{p} / Z_{p}\right) \rightarrow H^{0}\left(U, \mathbb{Q}_{p} / \mathbb{Z}_{p}\right)$ is the identity. By definition of $\eta_{G}$ and $\eta_{U}$ this implies the assertion.

\section{Set-Up}

We now want to deduce $\mathbb{Z}_{p}$-duality from these statements. This is most conveniently phrased on the triangulated level. We introduce ad-hoc a number of triangulated categories flexible enough to allow our constructions.

By $G$-module we mean a topological $\mathbb{Z}_{p}$-module with a continuous operation of $G$.

Definition 3.1. Let $G$ be a $p$-adic Lie group or a Poincaré group.

1. Let $C\left(G, \mathbb{Z}_{p}\right)$ (resp. $C\left(G, \mathbb{Q}_{p}\right)$, resp. $C\left(G, \mathbb{Q}_{p} / \mathbb{Z}_{p}\right)$ ) be the category of complexes of $G$-modules quasi-isomorphic to a bounded complex whose terms are free $\mathbb{Z}_{p}$-modules of finite rank with a continuous operation of $G$ (respectively such modules tensored with $\mathbb{Q}_{p}$, resp. such modules tensored with $\left.\mathbb{Q}_{p} / \mathbb{Z}_{p}\right)$.

2. Let $D\left(G, \mathbb{Z}_{p}\right)$ (resp. $D\left(G, \mathbb{Q}_{p}\right)$, resp. $D\left(G, \mathbb{Q}_{p} / \mathbb{Z}_{p}\right)$ ) be its localization with respect to the class of quasi-isomorphisms. It is triangulated.

3. In the case $G=\{e\}$, we write $D\left(\mathbb{Z}_{p}\right)$ (resp. $D\left(\mathbb{Q}_{p}\right)$, resp. $D\left(\mathbb{Q}_{p} / \mathbb{Z}_{p}\right)$ ). 
Note that $D\left(\mathbb{Z}_{p}\right)$ is the bounded derived category of finitely generated $\mathbb{Z}_{p}$-modules. All bounded complexes of finite dimensional $\mathbb{Q}_{p}$-vector spaces with continuous operation of $G$ are objects of $D\left(G, \mathbb{Q}_{p}\right)$ because such a vector space has a $G$-stable $\mathbb{Z}_{p}$-lattice.

As free $\mathbb{Z}_{p}$-modules are flat, there are triangulated functors

$$
\begin{aligned}
\cdot \otimes \mathbb{Q}_{p}: D\left(G, \mathbb{Z}_{p}\right) & \rightarrow D\left(G, \mathbb{Q}_{p}\right) \\
\cdot \otimes \mathbb{Q}_{p} / \mathbb{Z}_{p}: D\left(G, \mathbb{Z}_{p}\right) & \rightarrow D\left(G, \mathbb{Q}_{p} / \mathbb{Z}_{p}\right)
\end{aligned}
$$

As free $\mathbb{Z}_{p}$-modules are projective and $\mathbb{Q}_{p} / \mathbb{Z}_{p}$ is injective, there are triangulated functors

$$
\begin{gathered}
\cdot{ }^{\vee}=\operatorname{Hom}_{\mathbb{Z}_{p}}(\cdot, D): D\left(G, \mathbb{Z}_{p}\right) \rightarrow D\left(G, \mathbb{Z}_{p}\right) \\
\cdot{ }^{\vee}=\operatorname{Hom}_{\mathbb{Q}_{p}}\left(\cdot, D_{\mathbb{Q}_{p}}\right): D\left(G, \mathbb{Q}_{p}\right) \rightarrow D\left(G, \mathbb{Q}_{p}\right) \\
\cdot^{*}=\operatorname{Hom}_{\mathbb{Z}_{p}}\left(\cdot, D \otimes \mathbb{Q}_{p} / \mathbb{Z}_{p}\right): D\left(G, \mathbb{Z}_{p}\right) \rightarrow D\left(G, \mathbb{Q}_{p} / \mathbb{Z}_{p}\right) \\
\cdot^{*}=\operatorname{Hom}_{\mathbb{Z}_{p}}\left(\cdot D \otimes \mathbb{Q}_{p} / \mathbb{Z}_{p}\right): D\left(G, \mathbb{Q}_{p} / \mathbb{Z}_{p}\right) \rightarrow D\left(G, \mathbb{Z}_{p}\right)
\end{gathered}
$$

Lemma 3.2. There are commutative diagram of functors
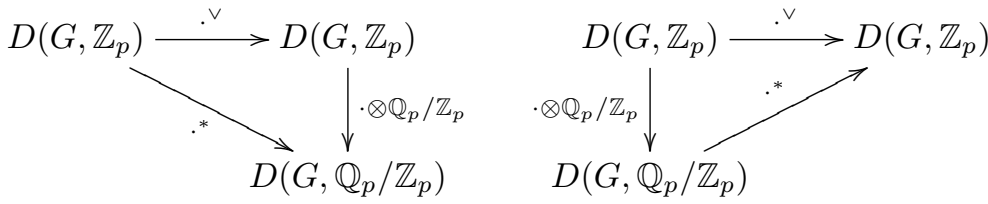

Proof. It suffices to consider the case of a single $G$-module $M$ which is free of finite rank over $\mathbb{Z}_{p}$. In this case the first statement says

$$
\operatorname{Hom}_{\mathbb{Z}_{p}}\left(M, \mathbb{Z}_{p}\right) \otimes \mathbb{Q}_{p} / \mathbb{Z}_{p} \cong \operatorname{Hom}_{\mathbb{Z}_{p}}\left(M, \mathbb{Q}_{p} / \mathbb{Z}_{p}\right)
$$

and the second

$$
\operatorname{Hom}_{\mathbb{Z}_{p}}\left(M, \mathbb{Z}_{p}\right) \cong \operatorname{Hom}_{\mathbb{Z}_{p}}\left(M \otimes \mathbb{Q}_{p} / \mathbb{Z}_{p}, \mathbb{Q}_{p} / \mathbb{Z}_{p}\right)
$$

Both are true.

\section{Group cohomology}

For a $G$-module $M$, let $C(G, M)$ be the continuous bar complex for $G$ with values in $M$, ie., the complex computing continuous group cohomology of $G$.

Lemma 4.1. Let $G$ be a Poincaré group. $C(G, \cdot)$ induces natural functors

$$
\begin{aligned}
& C(G, \cdot): D\left(G, \mathbb{Z}_{p}\right) \rightarrow D\left(\mathbb{Z}_{p}\right) \\
& C(G, \cdot): D\left(G, \mathbb{Q}_{p}\right) \rightarrow D\left(\mathbb{Q}_{p}\right) \\
& C(G, \cdot): D\left(G, \mathbb{Q}_{p} / \mathbb{Z}_{p}\right) \rightarrow D\left(\mathbb{Q}_{p} / \mathbb{Z}_{p}\right)
\end{aligned}
$$


Moreover, there are natural commuative diagrams of functors

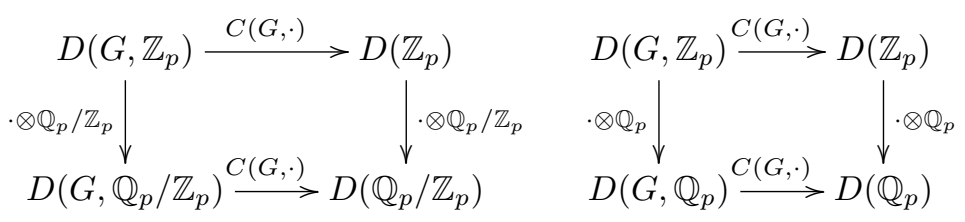

Proof. We first consider $D\left(G, \mathbb{Z}_{p}\right)$. The complexes $C(G, \cdot)$ are strictly functorial. Hence they extend immediately to complexes. Every short exact sequence of $G$-modules which are free of finite rank over $\mathbb{Z}_{p}$ gives rise to a long exact sequence in group cohomology. Hence $C(G, \cdot)$ extends to the triangulated setting. What we have to check are the finiteness conditions.

It suffices to consider complexes concentrated in degree 0 . Let $M$ be a $G$-module which is free and of finite rank as $\mathbb{Z}_{p}$-module. We have

$$
H^{i}(C(G, M))=H^{i}(G, M)=\lim _{\longleftarrow} H^{i}\left(G, M / p^{n} M\right)
$$

This vanishes for $i \notin[0, \ldots, d]$ because $G$ is a Poincaré group. As the cohomology group $H^{i}\left(G, M / p^{n} M\right)$ is finite and $p^{n}$-torsion, $H^{i}(G, M)$ is compact and hence of finite type as $\mathbb{Z}_{p}$-module. This implies that $C(G, M) \in D\left(\mathbb{Z}_{p}\right)$.

The statements for $D\left(G, \mathbb{Q}_{p}\right)$ and $D\left(G, \mathbb{Q}_{p} / \mathbb{Z}_{p}\right)$ follow from this once we have established the commutative diagrams. The commuative diagram for $D\left(G, \mathbb{Q}_{p}\right)$ is a standard assertion for continuous group cohomology. The diagram already commutes on the level of complexes. It follows by writing $\mathbb{Q}_{p}=\lim _{\longrightarrow} \frac{1}{p^{n}} \mathbb{Z}_{p}$ and from commutativity of continuous group cohomology with direct limits.

In the case of $D\left(G, \mathbb{Q}_{p} / \mathbb{Z}_{p}\right)$, write the derived functor $M \otimes \mathbb{Q}_{p} / \mathbb{Z}_{p}$ as the cone of $M \otimes \mathbb{Z}_{p} \rightarrow M \otimes \mathbb{Q}_{p}$. Then, again, the diagram already commutes on the level of complexes by the rational case.

Proposition 4.2. Let $G$ be a Poincaré group. There is a natural isomorphism of functors $D\left(G, \mathbb{Z}_{p}\right) \rightarrow D\left(\mathbb{Z}_{p}\right)$

$$
\cdot{ }^{\vee} \circ C(G, \cdot) \circ \cdot \vee \cong \cdot^{*} \circ C(G, \cdot) \circ \cdot *
$$

Proof. Put together the first commutative diagram of Lemma 3.2, the first diagram of Lemma 4.1 and the second diagram of Lemma 3.2 (for the group $\{e\})$ :

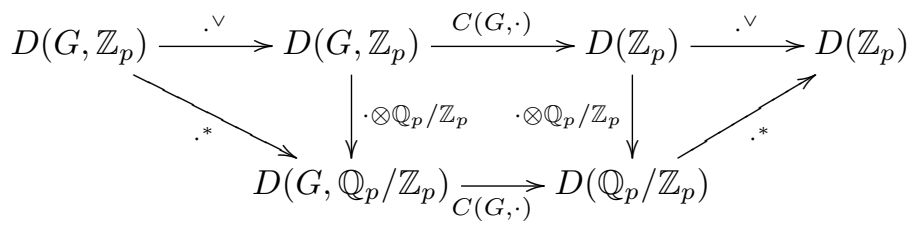




\section{Pairings}

Let $G$ be a Poincaré group of dimension $d$. The tensor product of complexes induces triangulated functors

$$
\begin{aligned}
& \otimes: D\left(G, \mathbb{Z}_{p}\right) \times D\left(G, \mathbb{Z}_{p}\right) \rightarrow D\left(G, \mathbb{Z}_{p}\right) \\
& \otimes: D\left(G, \mathbb{Z}_{p}\right) \times D\left(G, \mathbb{Q}_{p} / \mathbb{Z}_{p}\right) \rightarrow D\left(G, \mathbb{Q}_{p} / \mathbb{Z}_{p}\right)
\end{aligned}
$$

Let $M \in D\left(G, \mathbb{Z}_{p}\right)$. By adjunction there is a natural map $M \otimes M^{\vee} \rightarrow D$. Consider the cup-product pairing

$$
\begin{aligned}
C(G, M) \otimes C\left(G, M^{\vee}\right) \rightarrow C(G, M & \left.\otimes M^{\vee}\right) \\
& \rightarrow C(G, D) \rightarrow H^{d}(G, D)[-d] \cong \mathbb{Z}_{p}[-d]
\end{aligned}
$$

where the last isomorphism is given by the map $\eta: H^{d}(G, D) \rightarrow \mathbb{Z}_{p}$ defined in Section 2, (1) . By adjunction, it induces a morphism

$$
C(G, M) \rightarrow C\left(G, M^{\vee}\right)^{\vee}[-d]
$$

i.e, a transformation of functors

$$
\alpha: \mathrm{id} \rightarrow[-d] \circ \cdot^{\vee} \circ C(G, \cdot) \circ \cdot \vee
$$

Proposition 5.1. $\alpha$ is an isomorphism of functors.

Proof. Recall Pontrjagin duality. Let the transformation

$$
\beta: \mathrm{id} \rightarrow[-d] \circ \cdot^{*} \circ C(G, \cdot) \circ \cdot^{*}
$$

be induced by adjunction from the cup-product pairing

$$
\begin{aligned}
C(G, M) \otimes C\left(G, M^{*}\right) \rightarrow C(G, M \otimes & \left.M^{*}\right) \rightarrow C\left(G, D \otimes \mathbb{Q}_{p} / \mathbb{Z}_{p}\right) \\
& \rightarrow H^{d}\left(G, D \otimes \mathbb{Q}_{p} / \mathbb{Z}_{p}\right)[-d] \cong \mathbb{Z}_{p}[-d]
\end{aligned}
$$

with can : $H^{d}\left(G, D \otimes \mathbb{Q}_{p} / \mathbb{Z}_{p}\right) \cong \mathbb{Z}_{p}$ as in Section 2 induced by the universal property of $D$.

$\beta$ is known to be an isomorphism by Pontrjagin duality. Hence it suffices to show that $\alpha$ and $\beta$ are compatible under the isomorphism of Proposition 4.2. In order to do this, we rewrite the natural isomorphism of Lemma 4.1

$$
C(G, M) \otimes \mathbb{Q}_{p} / \mathbb{Z}_{p} \rightarrow C\left(G, M \otimes \mathbb{Q}_{p} / \mathbb{Z}_{p}\right)
$$

by using the cup-product pairing and the natural map

$$
\mathbb{Q}_{p} / Z_{p}=H^{0}\left(G, \mathbb{Q}_{p} / \mathbb{Z}_{p}\right) \rightarrow C\left(G, \mathbb{Q}_{p} / \mathbb{Z}_{p}\right)
$$

as

$$
C(G, M) \otimes \mathbb{Q}_{p} / \mathbb{Z}_{p} \rightarrow C(G, M) \otimes C\left(G, \mathbb{Q}_{p} / \mathbb{Z}_{p}\right) \rightarrow C\left(G, M \otimes \mathbb{Q}_{p} / \mathbb{Z}_{p}\right)
$$

In this description, the associativity of triple cup-products implies that the diagram

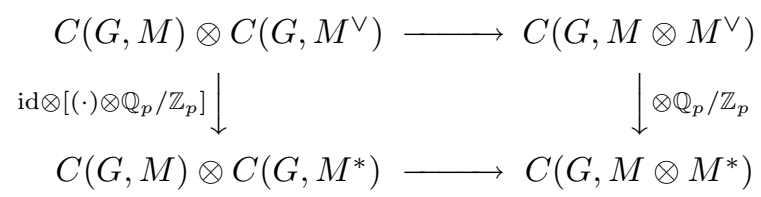


commutes in the derived category.

By simple functoriality, the diagram

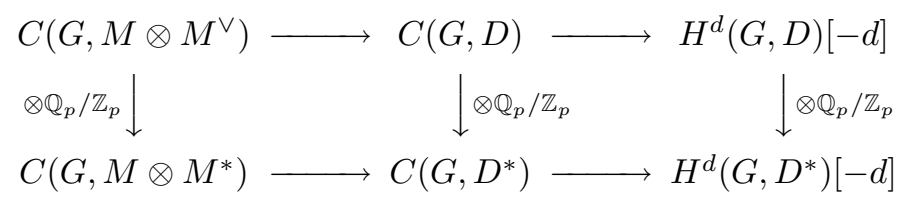

commutes also. Finally, recall that $\eta: H^{d}(G, D) \rightarrow \mathbb{Z}_{p}$ is the unique map such that the chain of isomorphisms

$$
\begin{aligned}
H^{d}\left(G, D^{*}\right) & \longleftarrow H^{d}(G, D) \otimes H^{0}(G, D) \otimes H^{0}\left(G, \mathbb{Q}_{p} / \mathbb{Z}_{p}\right) \\
& \stackrel{\eta \otimes \mathrm{id}}{\longrightarrow} \mathbb{Z}_{p} \otimes H^{0}\left(G, \mathbb{Q}_{p} / \mathbb{Z}_{p}\right) \rightarrow \mathbb{Q}_{p} / \mathbb{Z}_{p}
\end{aligned}
$$

is given by the canical isomorphism can. In other word, in our reinterpretation of $\cdot \otimes \mathbb{Q}_{p} / \mathbb{Z}_{p}$, the diagram

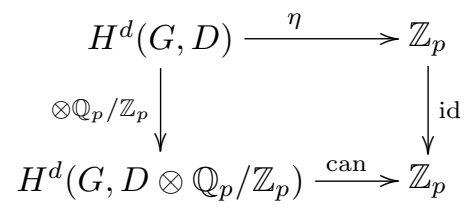

commutes.

Putting together these three diagrams we get

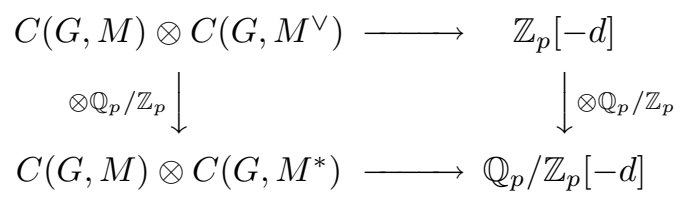

By adjunction, the first line defines $\alpha$ and the second defines $\beta$. Hence they are compatible.

\section{Rational Poincaré duality}

Let $G$ be $p$-adic Lie group of dimension $d$.

Lemma 6.1 (Lazard). There is an open subgroup $U \subset G$ which is a Poincaré group of dimension $d$. The dualizing module $D$ is the free $\mathbb{Z}_{p}$-module of rank 1 with the operation of $G$ given by multiplication by $\chi(x)=\operatorname{det} \operatorname{Ad}(x)$.

Proof. $G$ contains an open subgroup $G^{\prime}$ which is pro-p. By [L] V 2.2.7.1 $G^{\prime}$ contains an open subgroup $U$ which is equi- $p$-valued (for a suitable filtration). By loc.cit. V Theorem 2.5.7 this subgroup is a Poincaré group. By loc.cit. V Theorem 2.5.8. it has dimension $d$ and the dualizing module has the given shape. 
Definition 6.2. Let $G$ be a $p$-adic Lie group of dimension $d$. Let $D_{\mathbb{Q}_{p}}$ be the $\mathbb{Q}_{p}$-vector space of dimension 1 with operation of $G$ given by multiplication by $\chi(x)=\operatorname{det} \operatorname{Ad}(x)$.

Let $U$ be an open subgroup which is Poincaré. Put

$$
\eta=\frac{1}{(G: U)} \eta \circ \text { res }: H^{d}\left(G, D_{\mathbb{Q}_{p}}\right) \longrightarrow H^{d}\left(U, D_{\mathbb{Q}_{p}}\right) \rightarrow \mathbb{Q}_{p}
$$

By Lemma 2.1, this is well-defined.

Let $V \in D\left(G, \mathbb{Q}_{p}\right)$. By adjunction there is a natural map $V \otimes V^{\vee} \rightarrow D_{\mathbb{Q}_{p}}$. Consider the cup-product pairing

$$
\begin{aligned}
C(G, V) \otimes C\left(G, V^{\vee}\right) \rightarrow C\left(G, V \otimes V^{\vee}\right) & \rightarrow C\left(G, D_{\mathbb{Q}_{p}}\right) \\
& \rightarrow H^{d}\left(G, D_{\mathbb{Q}_{p}}\right)[-d] \stackrel{\eta}{\rightarrow} \mathbb{Q}_{p}[-d]
\end{aligned}
$$

By adjunction it induces a natural transformation

$$
\alpha: C(G, V) \rightarrow C\left(G, V^{\vee}\right)^{\vee}[-d]
$$

for all $V \in D\left(G, \mathbb{Q}_{p}\right)$.

Proposition 6.3. $\alpha$ is an isomorphism.

Proof. If $G$ is a Poincaré group, the transformation is compatible with the one in Proposition 5.1 under extension of scalars. In particular, it is an isomorphism.

For general $G$, it suffices to consider the case of a complex concentrated in degree 0 . Let $V=M \otimes \mathbb{Q}_{p}$ be such an object. The claim is equivalent to saying that the pairing

$$
<\cdot, \cdot>: H^{i}(G, V) \times H^{d-i}\left(G, V^{\vee}\right) \rightarrow H^{d}(G, D) \stackrel{\eta}{\rightarrow} \mathbb{Q}_{p}
$$

is perfect. Let $U$ be a subgroup which is Poincaré. Note that $H^{i}(G, V)$ is a direct summand of $H^{i}(U, V)$ via the restriction map because cores $\circ$ res = $(G: U)([\mathrm{NSW}]$ Ch. I $\S 5$ Corollary 1.5.7). The pairing for $U$ and $\operatorname{res}(V)$ is perfect by the first case. The projection formula

$$
<x, \operatorname{res}(y)>_{U}=<\operatorname{cores}(x), y>_{G}
$$

holds by [NSW] Ch. I $\S 5$ Proposition 1.5.3 and our choice or normaliziation of $\eta$. This implies the statement for $G$.

We have established Poincaré duality with rational coefficients, i.e, Theorem 1.1 has just been proved. (Note that any finite dimensional $\mathbb{Q}_{p}$-vector space $V$ with continuous operation of $G$ is of the form $M \otimes \mathbb{Q}_{p}$ for a free $\mathbb{Z}_{p}$-module of finite rank).

The promised integral structures are given by the duality on $D\left(G, \mathbb{Z}_{p}\right)$ of Proposition 5.1. By construction it is compatible with the one on $D\left(G, \mathbb{Q}_{p}\right)$.

Proof of Theorem 1.2. As in the last proof, by the projection formula and our normalization of $\eta$ it suffices to consider the case $G$ a Poincaré group.

Let $M=D, M^{\vee}=\mathbb{Z}_{p}$. We evaluate the duality of Proposition 5.1

$$
H^{d}(\alpha): H^{d}(G, D) \cong H^{0}\left(\operatorname{Hom}_{\mathbb{Z}_{p}}\left(C\left(G, \mathbb{Z}_{p}\right), \mathbb{Z}_{p}\right)\right)=\operatorname{Hom}_{D\left(\mathbb{Z}_{p}\right)}\left(C\left(G, \mathbb{Z}_{p}\right), \mathbb{Z}_{p}\right)
$$


As the cohomological dimension of $\mathbb{Z}_{p}$ is 1 , we have

$$
\begin{aligned}
0 \rightarrow \operatorname{Ext}_{\mathbb{Z}_{p}}^{1}\left(H^{1}\left(G, \mathbb{Z}_{p}\right), \mathbb{Z}_{p}\right) \rightarrow \operatorname{Hom}_{D\left(\mathbb{Z}_{p}\right)}\left(C\left(G, \mathbb{Z}_{p}\right), \mathbb{Z}_{p}\right) \\
\rightarrow \operatorname{Hom}_{\mathbb{Z}_{p}}\left(H^{0}\left(G, \mathbb{Z}_{p}\right), \mathbb{Z}_{p}\right) \rightarrow 0
\end{aligned}
$$

We claim that the first term vanishes. A priori, it is $p$-torsion. On the other hand, by Pontrjagin duality, $H^{d}(G, D) \cong H^{0}\left(G, \mathbb{Q}_{p} / \mathbb{Z}_{p}\right)^{*} \cong \mathbb{Z}_{p}$ is torsion free. Hence the middle group cannot have a torsion subgroup. This implies that

$$
H^{d}(G, D) \cong \operatorname{Hom}_{D\left(\mathbb{Z}_{p}\right)}\left(C\left(G, \mathbb{Z}_{p}\right), \mathbb{Z}_{p}\right) \cong \operatorname{Hom}\left(H^{0}\left(G, \mathbb{Z}_{p}\right), \mathbb{Z}_{p}\right)
$$

This compatibility of the integral structures under the Poincaré duality pairing proves Theorem 1.2.

Remark 6.4. For those $p$-adic Lie groups which are $p$-valued for some choice of filtration, there is by [L] $\mathrm{V}$ Theorem 2.4 .9 (see also [SW] Theorem 5.2.2) an isomorphism between $H^{i}(G, V)$ and $H^{i}(L, V)$ (where $L$ is the Lie algebra of $G$ ). As Lie algebra cohomology also satisfies Poincaré duality, this gives a different approach to proving Theorem 1.1. In [HKN] we have been able to control integral structures under this ismorphism for a more restrictive category of groups. Theorem 1.2 is weaker, but more general and a lot easier to prove.

\section{Acknowledgment}

I would like to thank the anonymous referee for his or her comments, making this note more correct than it would have been.

\section{References}

[HK] A. Huber, G. Kings, A cohomological Tamagawa number formula. preprint 2009, to appear Nagoya Math. J.

[HKN] A. Huber, G. Kings, N. Naumann, Some complements to the Lazard isomorphism. Preprint 2009, to appear Compositio Math.

[L] M. Lazard, Groupes analytiques p-adiques. Publ. IHES No. 26, 1965.

[N] J. Nekovar, Selmer complexes. Astérisque 310, Soc. Math. de France, 2006.

[NSW] J. Neukirch, A. Schmidt, K. Wingberg, Cohomology of number fields. Grundlehren der Mathematischen Wissenschaften 323. Springer-Verlag, Berlin, 2000.

[Se] J.-P. Serre, Cohomologie galoisienne. Lecture Notes in Mathematics, No. 5. Troisime dition, 1965 Springer-Verlag, Berlin-New York 1965.

[SW] P. Symonds, T. Weigel, Cohomology of p-adic analytic groups. New horizons in pro- $p$ groups, 349-410, Progr. Math., 184, Birkhuser Boston, Boston, MA, 2000. 
Annette Huber

Annette HuberrMathematisches Institut

Universtität Freiburg

Eckerstr. 1

79102 Freiburg

Germany

e-mail: annette.huber@math.uni-freiburg.de 